\title{
Monitoring of land cover change by citizens: The FotoQuest experience
}

Juan Carlos Laso Bayas, Linda See, Tobias Sturn, Mathias Karner, Dilek Fraisl, Inian Moorthy, Anto Subash, Ivelina Georgieva, Gerid Hager, Myroslava Lesiv, Hadi Hadi, Olha Danylo, Santosh Karanam, Martina Dürauer, Domian Dahlia, Dmitry Shchepashchenko, Ian McCallum, and Steffen Fritz

GEO_- Center for Earth Observation and Citizen Science (EOCS)

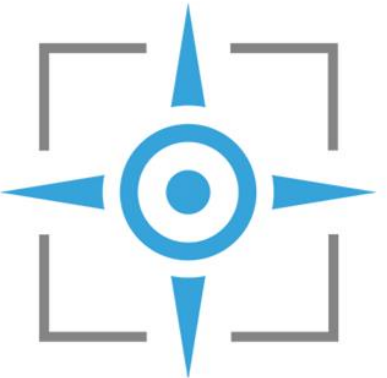

FotoQuestGo Europe 2018 


\section{Hello and welcome!}

In these slides you will find a short description and comparison of the 2 FotoQuest campaigns (2015 and 2018) against Eurostat LUCAS.

The slides describe how the FotoQuest 2015 and 2018 interfaces and campaigns looked like. In the final slides, initial analyses comparing campaign results will be shown, but If you cannot wait for that here it is:

\section{FotoQuest 2018 did much better than 2015, and it keeps improving!}

Please enjoy and direct any questions to Juan Laso lasobaya@iiasa.ac.at 
FotoQuest is a mobile app for citizens

to report land use and land cover at specific locations

The selected locations match LUCAS:

a Eurostat 3-yearly survey done by paid surveyors across Europe

\section{FotoQuest interface mimics LUCAS protocol}

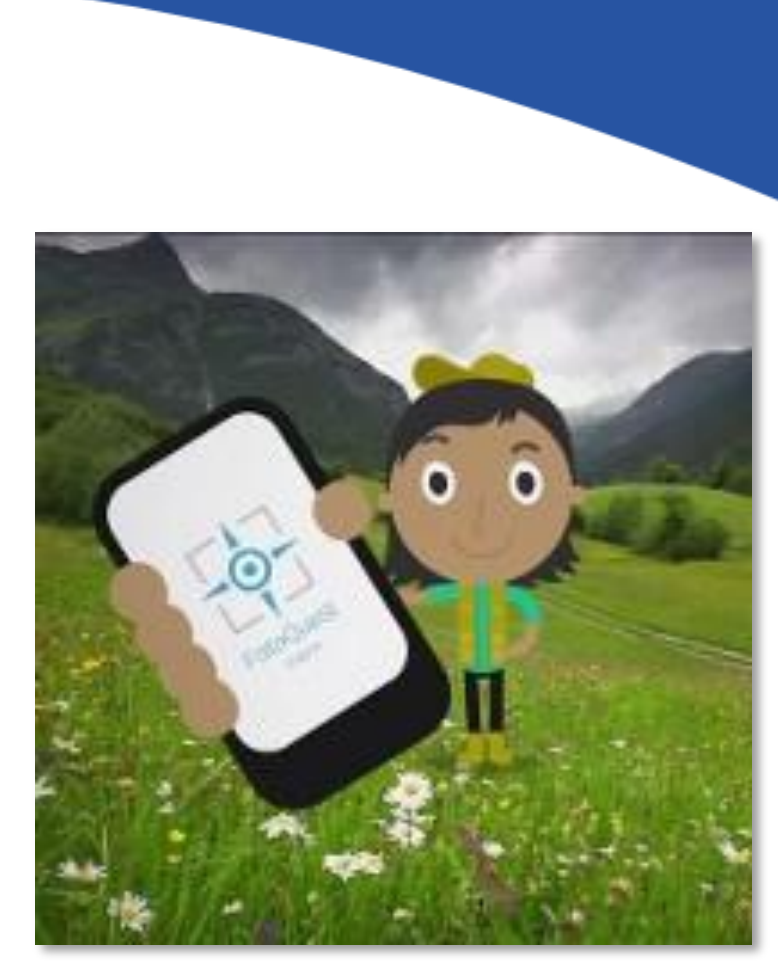

....it tries to understand citizens' land cover/use reporting accuracy

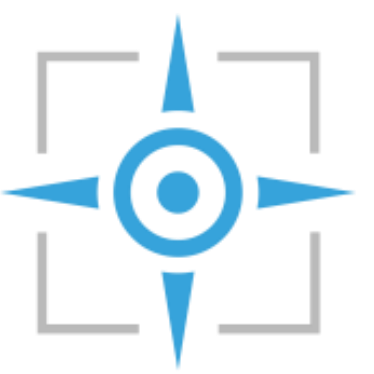

FotoQuest
FotoQuest initial campaign: 2015, in Austria.

Intermediate campaign: 2017 - Austria (to test new features).

FotoQuest Europe-wide campaign: 2018

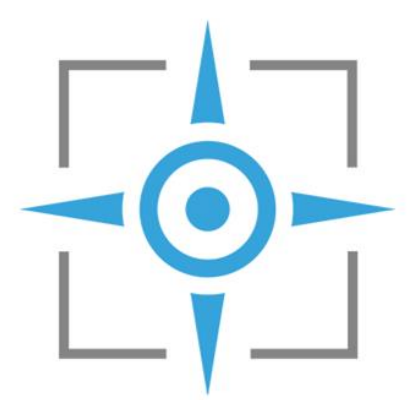

FotoQuestGo

Europe 2018 


\section{FotoQuest uses the mobile phone GPS, compass and camera}

\section{capabilities to guide participants to the selected}

locations

In both campaigns, the app asked and guided the user to take pictures in the four cardinal directions and of the location visited

It also only allowed to take pictures only once a user was close enough to the location or when an obstacle impeded access. It registered custom reasons such as "in private property"

- Take photos of the landscape. Two-thirds of a picture should show the ground and one-third the sky.

- When taking photos when there is an obstruction (wall, building, hedgerow, etc.) just keep your device horizontal and disregard the rule above.

- As much as possible, avoid identifications of persons or property while taking the pictures (e.g. car identification plates or people's faces). You can also blur out parts of the images later.

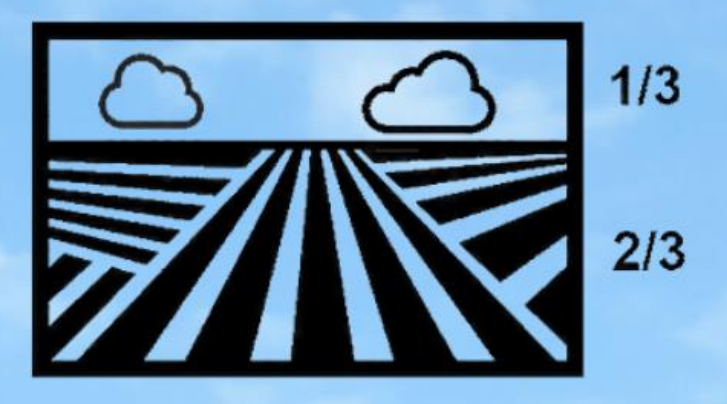

\section{Ok}




\section{FotoQuest: Picture taking}

\section{$\underline{\text { In } 2015}$}
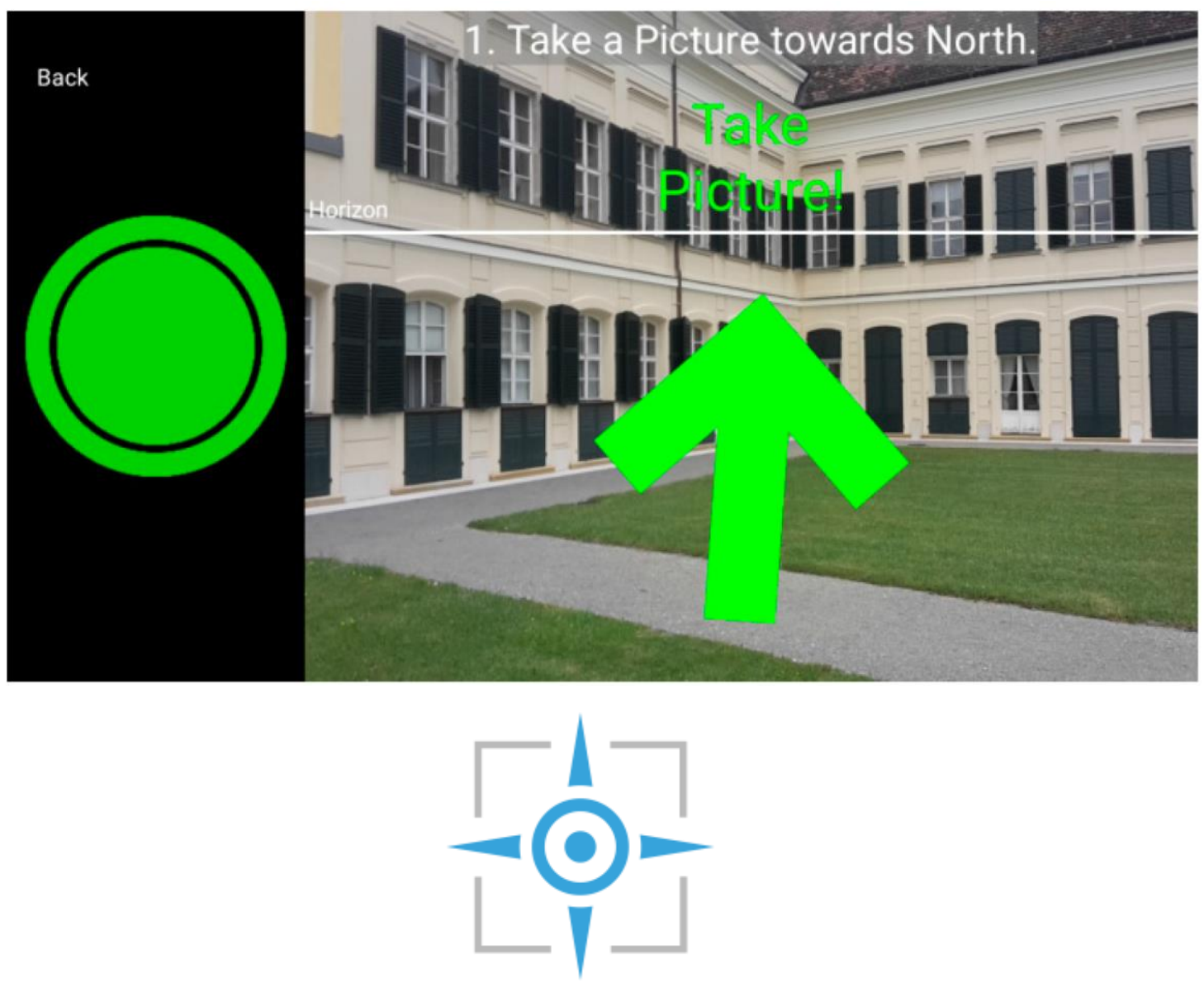

FotoQuest

\section{$\underline{\text { In } 2018}$}
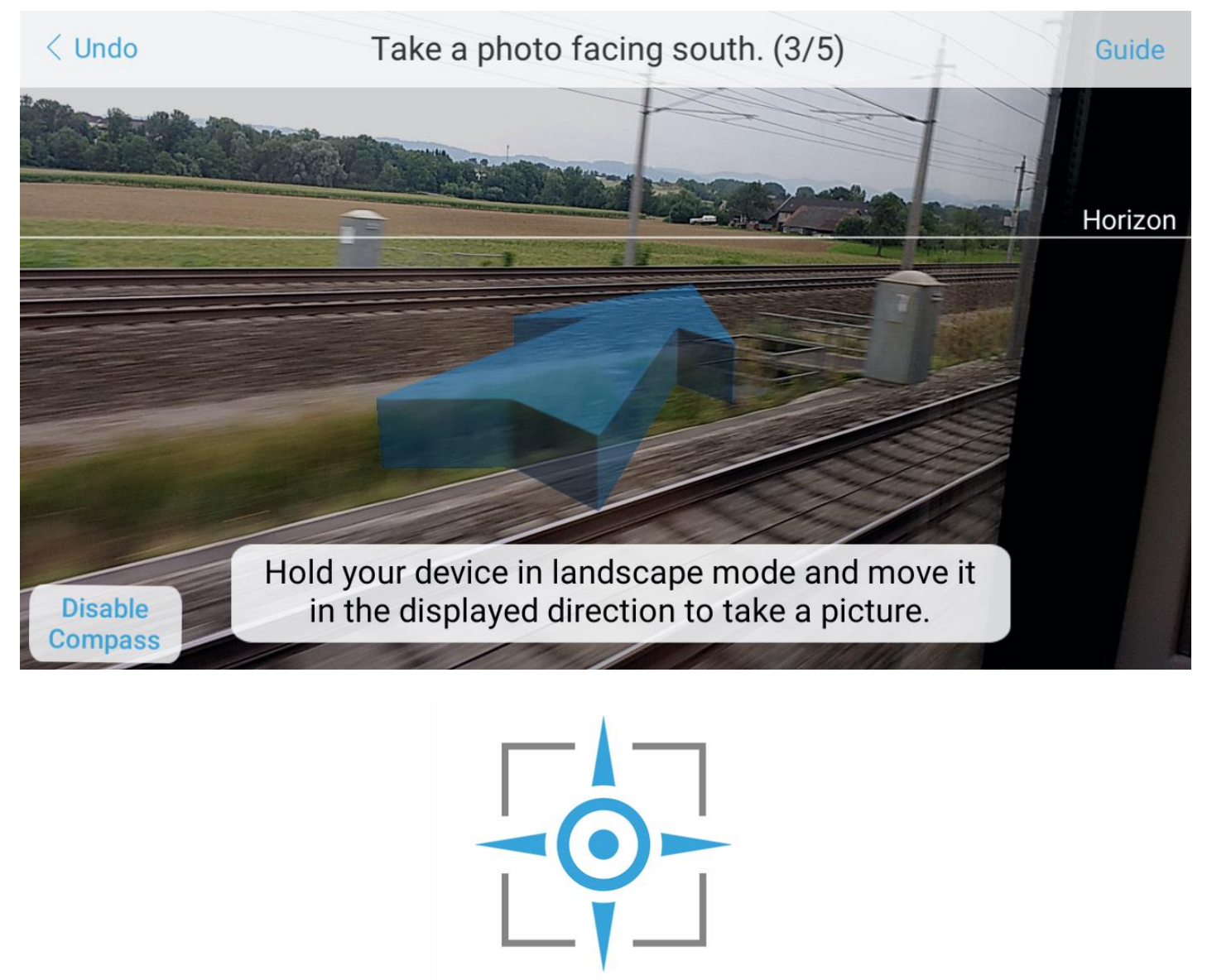

FotoQuestGo

Europe 


\section{FotoQuest Austria 2015: Interface}
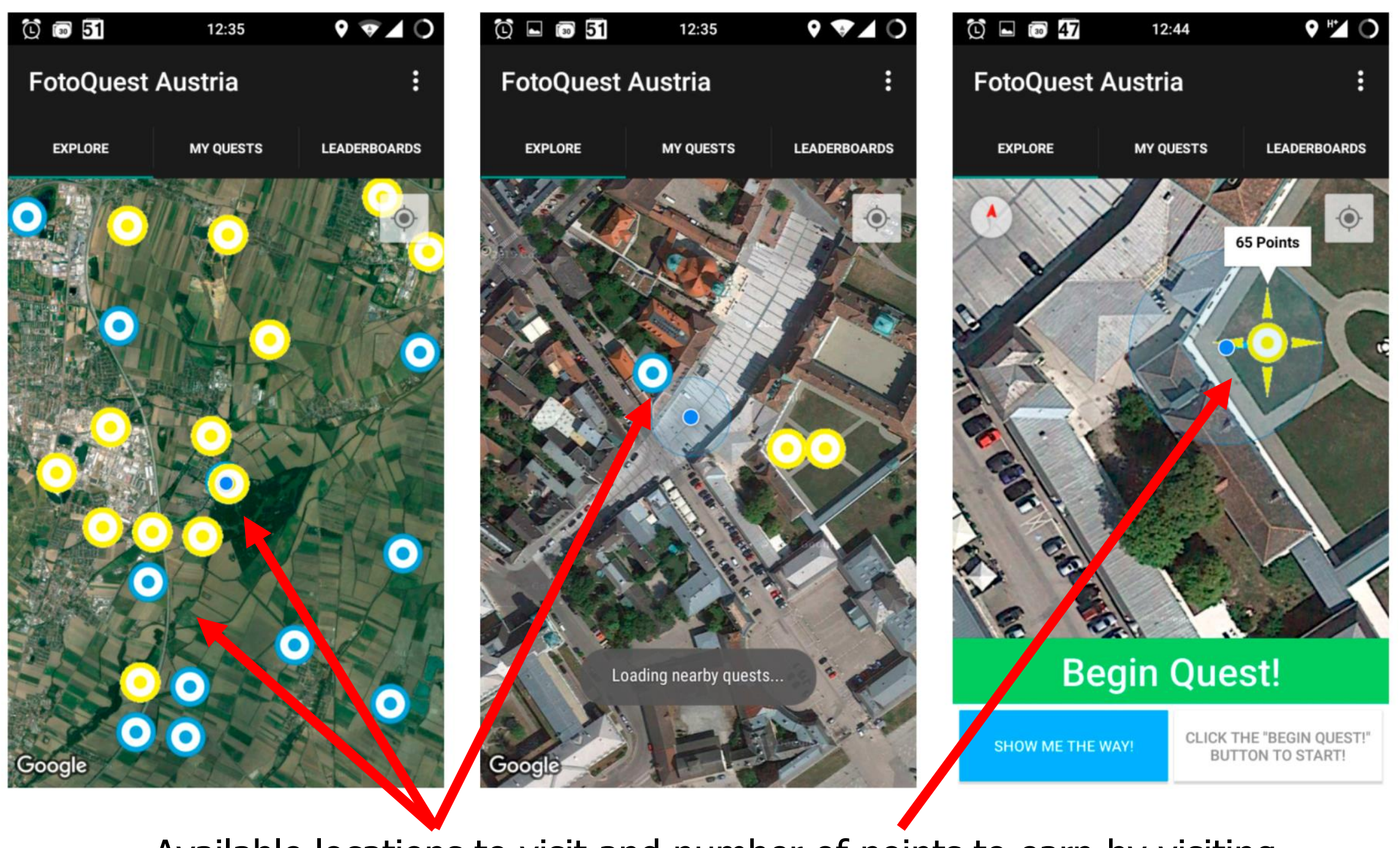

Available locations to visit and number of points to earn by visiting (Figure source: adapted from Laso Bayas et al. 2016, Remote Sensing) 


\section{FotoQuest Austria 2015: \\ Land cover selection}
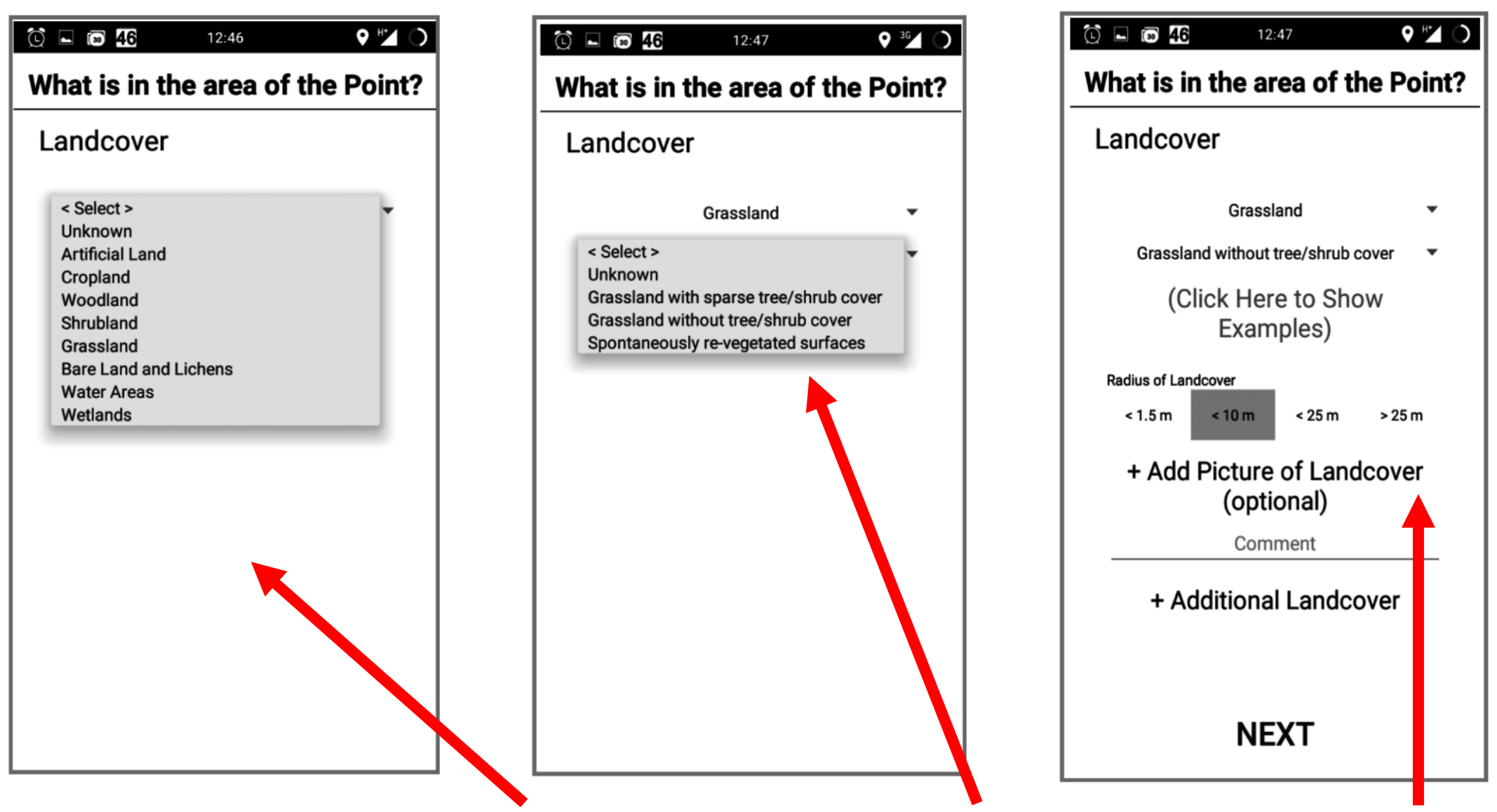

Land cover selection at level 1, corresponding level 2 plus additional options

(Figure source: adapted from Laso Bayas et al. 2016, Remote Sensing)

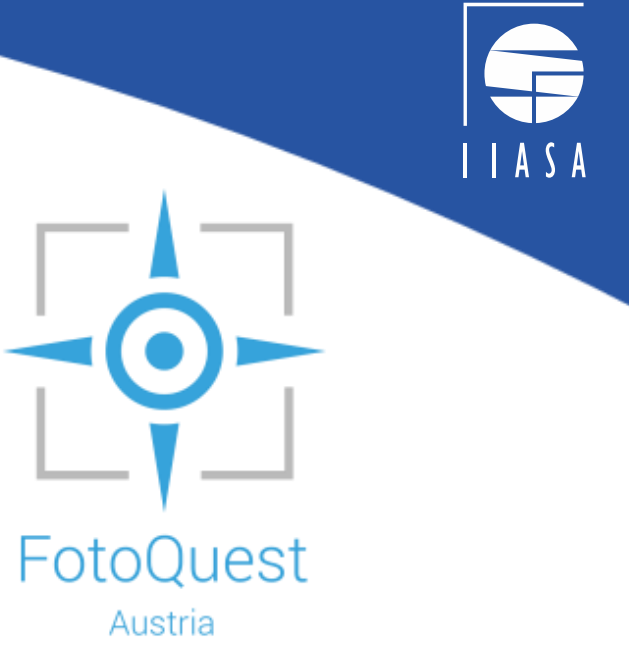

After taking pictures, users were asked to select land cover (and land use) at different levels, homogeneity radius of the observed land cover and could add additional land cover choices 


\section{FotoQuest Go Europe 2018: Interface}

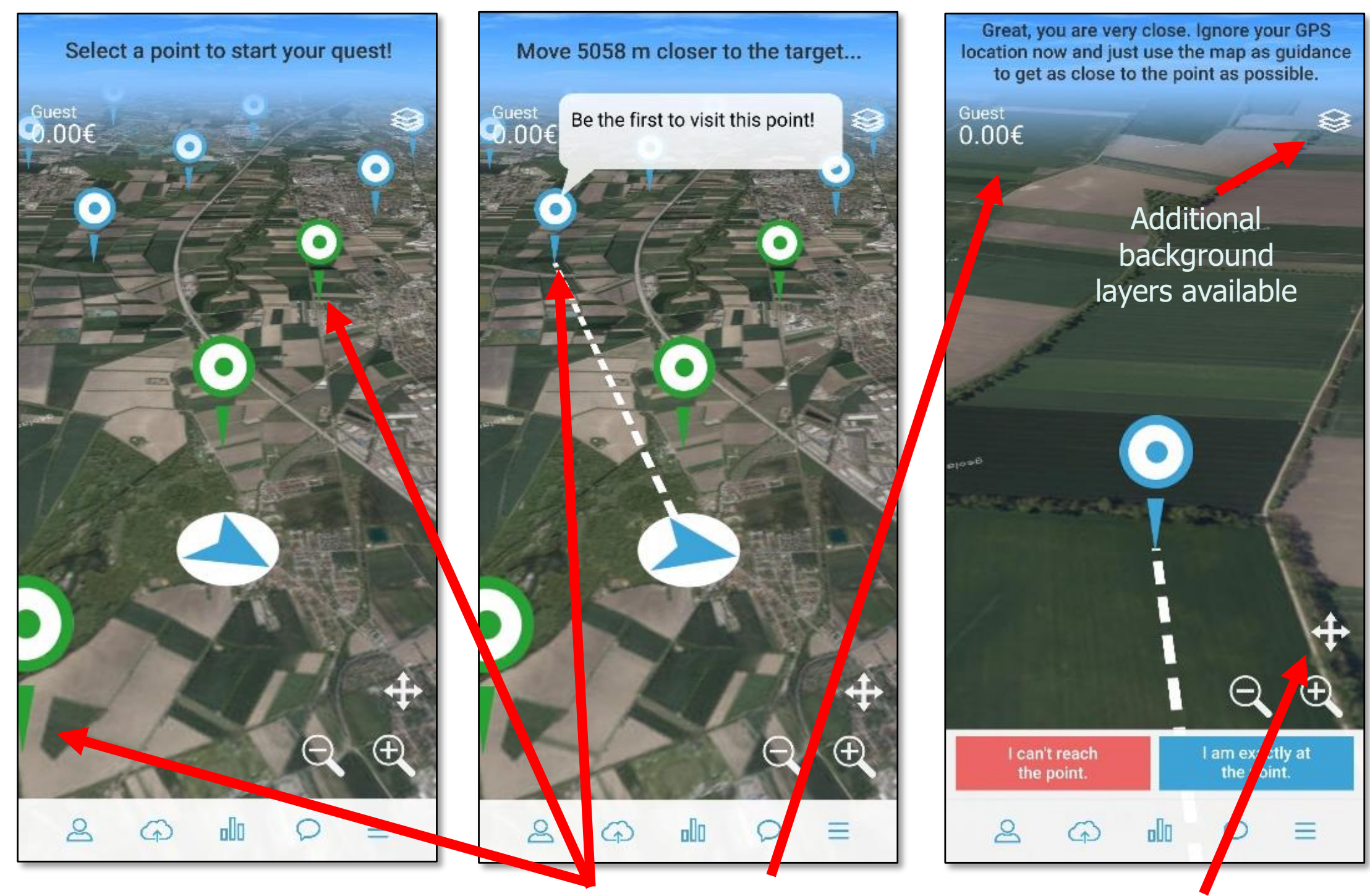

Available locations to visit, money $(€)$ earned, and 2D/3D map view

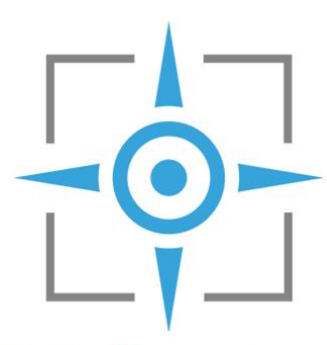

FotoQuestGo Europe

\section{Rewards:}

Each location visited awarded the participant between 1 to 3 Euros, depending on the level of difficulty, e.g. $€ 3$ for visiting points on sites far from roads

Weekly challenges with 1 random point awarded $\boldsymbol{€} \mathbf{3 0}$ Euros to the first visitor 


\section{FotoQuest Go Europe 2018: Change} detection

In 2018, users were first asked to determine if any change had happened

They were shown LUCAS 2015 pictures from each corresponding location.

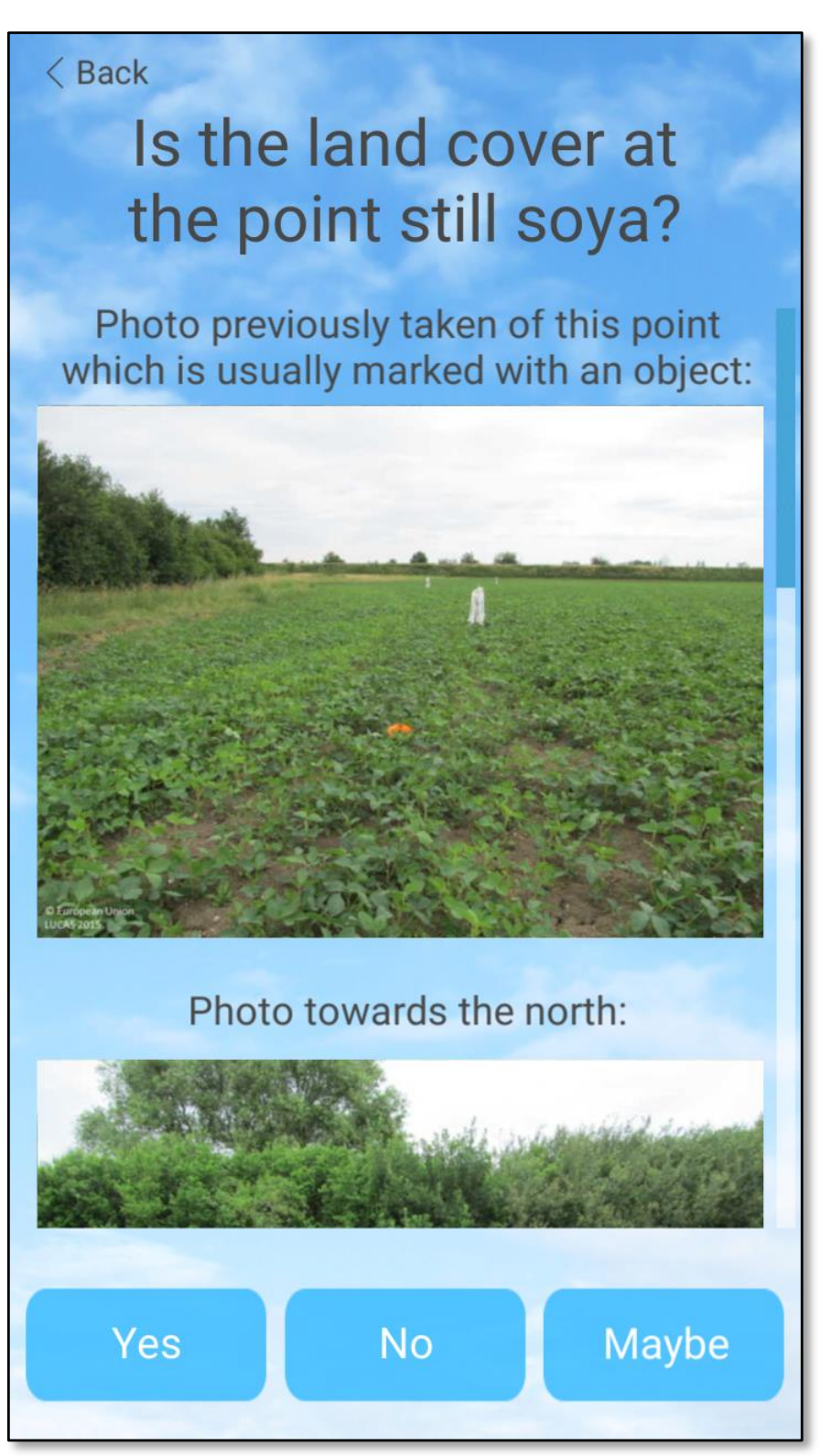




\section{FotoQuest Go Europe 2018: Land cover selection}

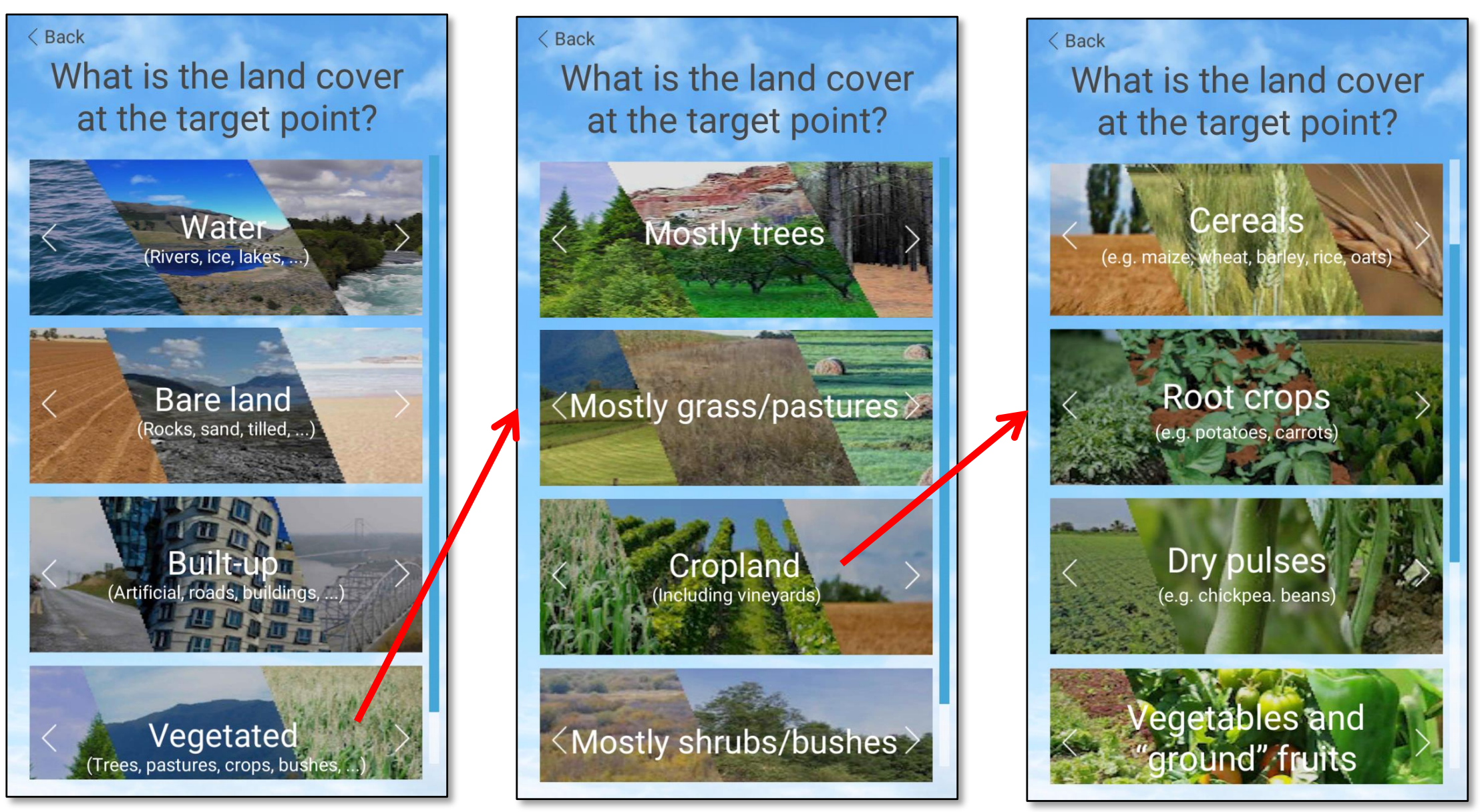

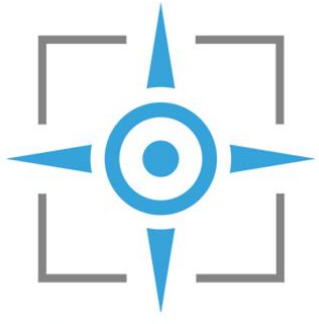

FotoQuestGo

Europe

In 2018, whether change or no change was selected, users were asked to take pictures.

Those that selected change were asked to select land cover at different levels, using picture-guided decision trees

Land cover selection at level 1, corresponding level 2, and 3 sequentially 


\section{FotoQuest Go Europe 2018:}

\section{Homogeneity and land use selection}
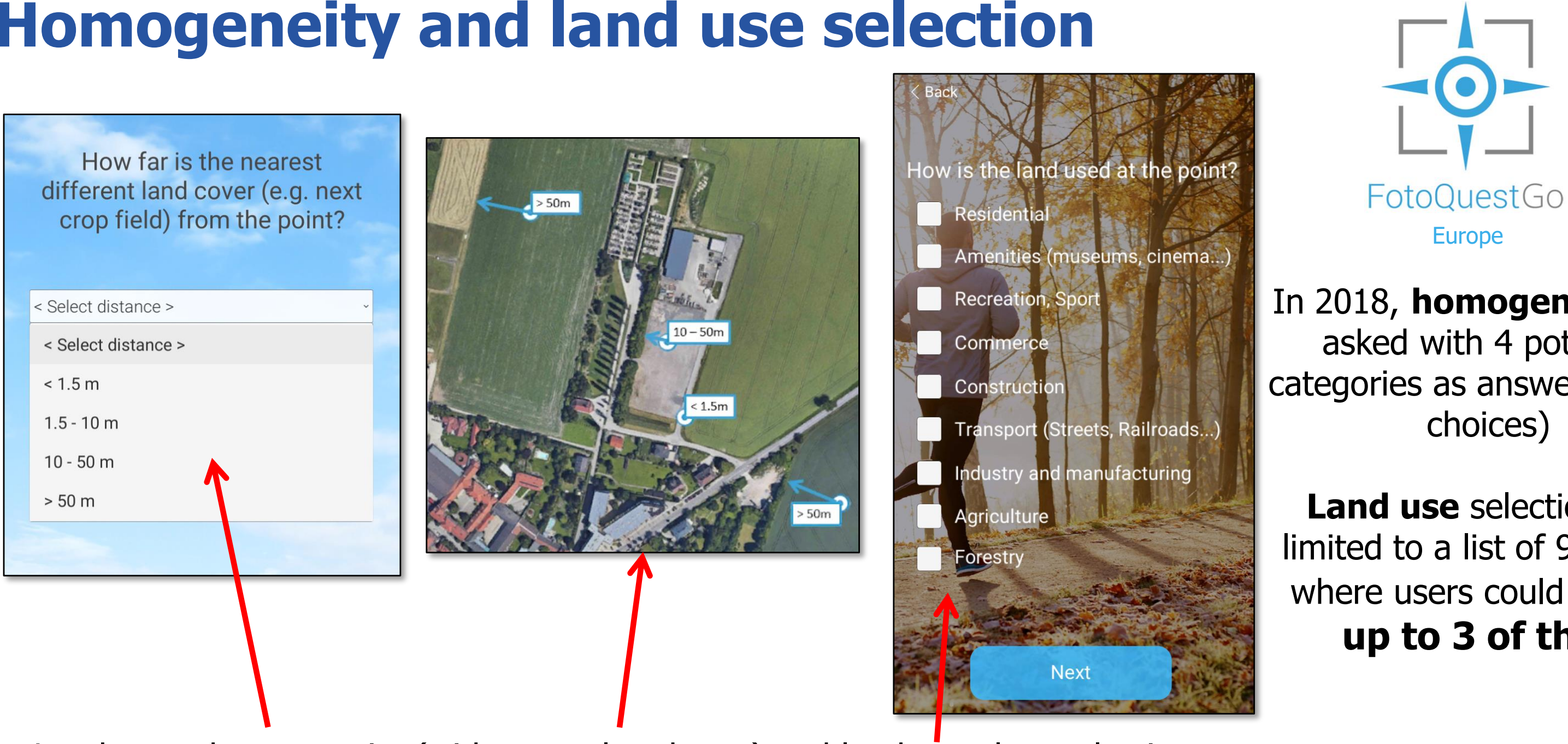

FotoQuestGo

Europe

In 2018, homogeneity was

asked with 4 potential categories as answers (range choices)

Land use selection was limited to a list of 9 options where users could select up to 3 of them

Land cover homogeneity (with examples shown) and land use class selection 


\section{FotoQuest Go Europe 2018: NRT-}

\section{Feedback}

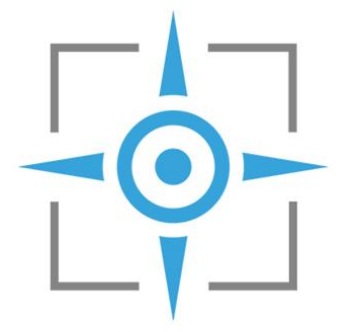

FotoQuestGo

Europe

In 2018, a near real time system providing users feedback in $\mathbf{1}$ day or less on all submitted quests ran during the campaign.

The system was tested on the 2017 intermediate campaign

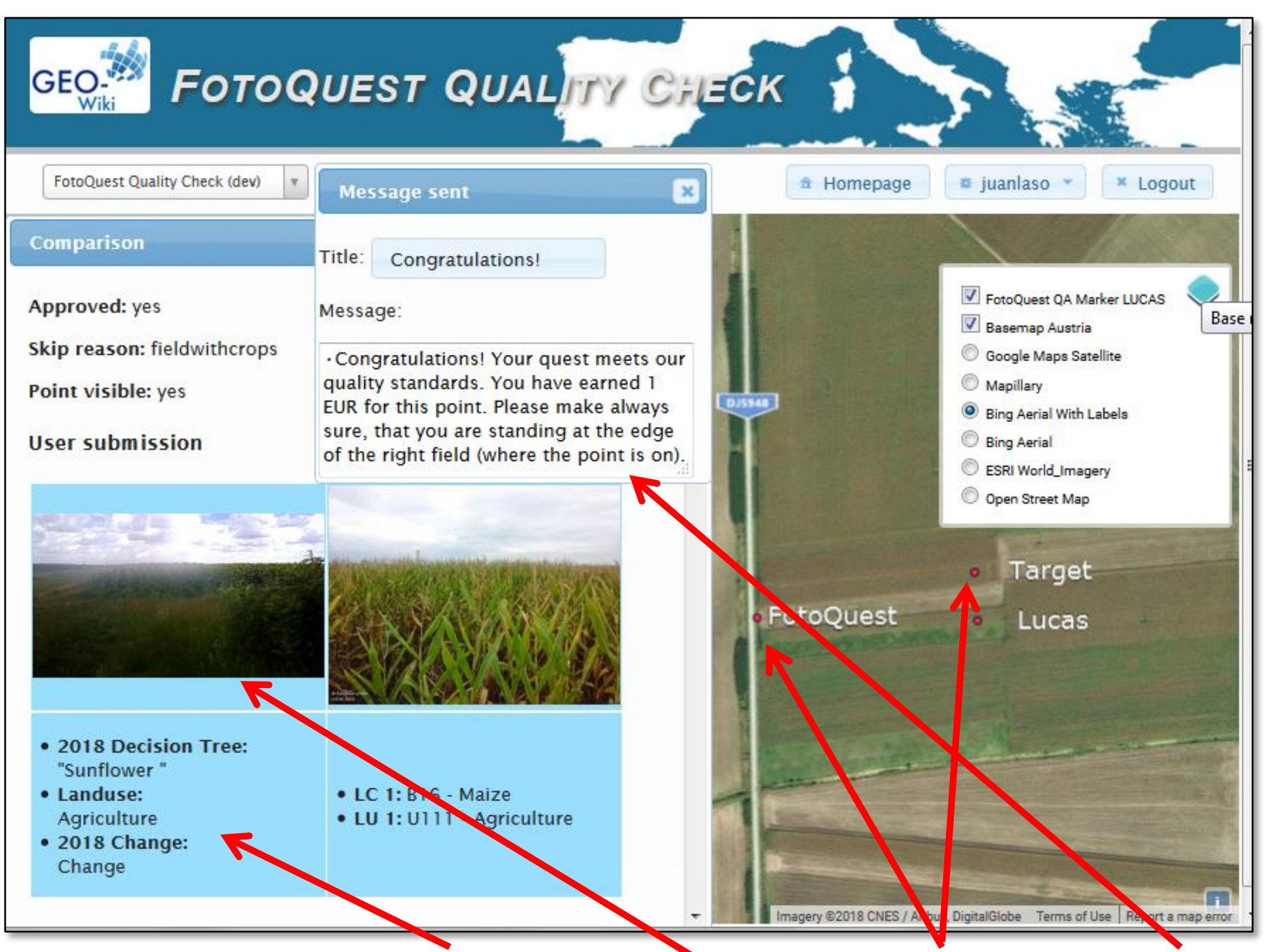

Geo-Wiki branch to visualize quests, compare pictures and distance, and send feedback 


\section{Summary: Campaigns characteristics compared}

2015:

- Only in Austria, quest could be visited more than once

- Prizes awarded at the end of the campaign, based on points/scoreboard

- Land cover decision tree not very user friendly nor guiding users

- No added layers and guides on the map

- No reference to past land cover status

- No near real-time feedback although users could communicate with IIASA

- Massive media campaign
2018:

- Europe-wide, quest can be visited only once

- Training was provided as videos accessible online

- Rewards were immediate, based upon approval from near real-time quality review

- User friendly graphical land cover decision-tree with mutually excluding hierarchical choices

- Several auxiliary map layers available

- Change detection: LUCAS 2015 pictures shown as reference

- Near real-time feedback and quality control

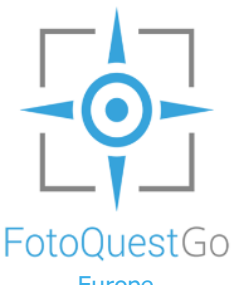




\section{Initial results compared}
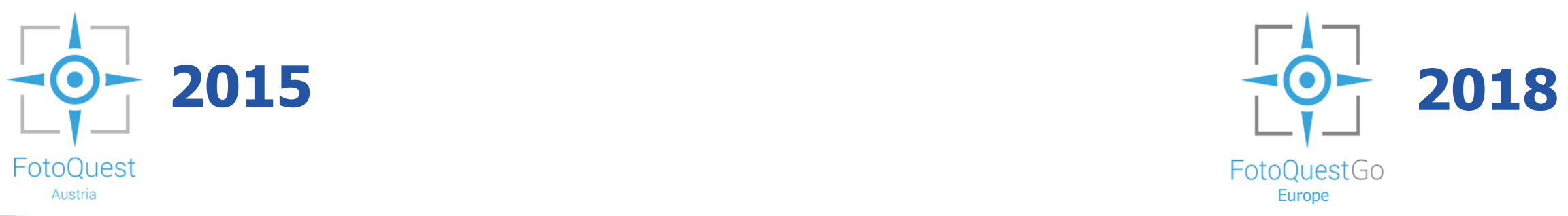

76

Only Austria

1699

( 300 used for analyses)

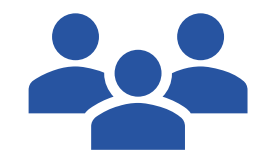

Number of users

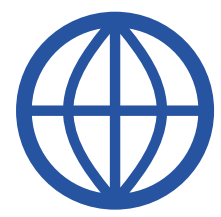

Geographical reach

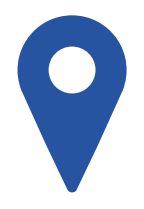

Number of unique locations
140

18 EU countries

1612

( 700 used for analyses) 


\section{Initial results compared}

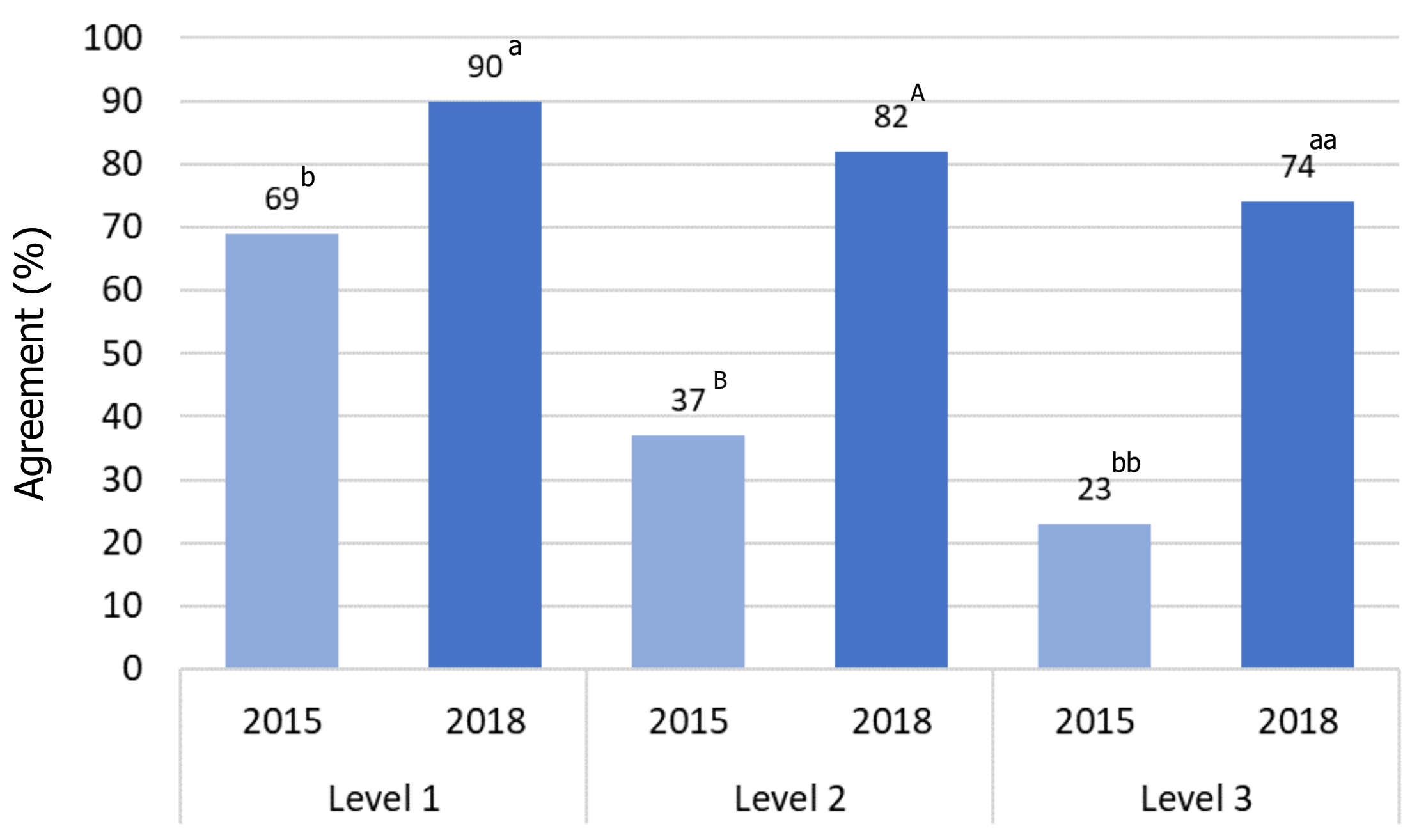

Land cover levels and FotoQuest campaigns
Land cover classifications done by the $\mathbf{2 0 1 8}$

FotoQuest participants were $\mathbf{2 . 9}$ to 3.5 times more likely to agree with LUCAS survey results than those of the 2015

(Cochran-NGafmpladiglel tests, $\left.\mathrm{p}<0.001, \mathrm{n}_{1}=1006, \mathrm{n}_{2}=955, \mathrm{n}_{3}=696\right)$ 


\section{Initial conclusions and current work}

- Improved protocol, visual guidance and choice restrictions (change/no change) approach allowed 2018 participants to have higher accuracy than 2015 participants

- Near real-time feedback allowed some degree of quality control although more work is needed to separate effects: Participants were encouraged to participate despite sometimes not having high quality quests

- People are motivated to participate, but promotion and support is needed for massive uptake

- Cost analysis and full description of 2018 campaign (e.g. accuracy per class, effects of feedback) is being performed, but costs are clearly lower with very high quality results. 


\section{More information on the FotoQuest Austria 2015:}

\section{Laso Bayas, J. C. et al. Crowdsourcing In-Situ Data on Land Cover and Land Use Using Gamification and Mobile Technology. Remote Sens. 8, e905 (2016)}

Watch out soon for a full journal article describing the 2018 campaign and its results!

Thanks to Michaela Busch for her support providing NRT Feedback to participants

This work was supported by the EU FP7 funded ERC grant Crowdland (No. 617754) 


\section{Hope you enjoyed!}

Please write your questions on the live chat session 